\title{
BENEFÍCIOS E PROBLEMAS RELACIONADOS AO USO DE PEUMUS BOLDUS (BOLDO-DO-CHILE) COMO ALTERNATIVA TERAPÊUTICA
}

\author{
BENEFITS AND PROBLEMS RELATED TO THE USE OF PEUMUS \\ BOLDUS (BOLDO-DO-CHILE) AS A THERAPEUTIC ALTERNATIVE
}

\begin{abstract}
Maísa Schneider Lazarotto ${ }^{1}$, Andressa Pagno ${ }^{1}$, Tainá Monique Schneider ${ }^{1}$, Talitta Copetti ${ }^{1}$

${ }^{1}$ Universidade Integrada Do Alto Uruguai e Das Missóes - URI Santo Ângelo, Santo Ângelo, Rio Grande do Sul, Brasil.
\end{abstract}

\section{RESUMO}

As práticas com plantas medicinais são populares no mundo inteiro, em alguns lugares elas são utilizadas com mais frequência, principalmente quando se trata da cultura de determinada comunidade. Peumus boldus, ou boldo como é popularmente conhecido, é comumente encontrado nos quintais de casas ou até mesmo na forma industrializada. Este trabalho trata-se de uma revisão bibliográfica, realizada no período de agosto a novembro de 2019. Na qual observou-se o uso do boldo e seus benefícios e problemas como alternativa terapêutica. A pesquisa buscou por estudos publicados do ano de 2009 à 2019, tendo como critérios de inclusão artigos originais, apenas na língua portuguesa, que apresentassem estudos com o boldo da espécie Peumus boldus, que falassem sobre a utilização de plantas e para qual finalidade terapêutica. Peumus boldus tem posição de destaque, relacionado à predominância de uso, e que, quando consumido de forma incorreta, pode causar má formação fetal, diarreia, entre outros. No entanto, quando é consumido de forma correta, traz muitos benefícios para algumas alteraçôes gastrointestinais, como por exemplo, age como antiflatulento, hepatoprotetor, digestivo, etc. Portanto, é importante que sejam conhecidos, pela população pelos profissionais da área da saúde, os benefícios e também os problemas do uso de plantas medicinais, em especial o boldo.

Descritores: Plantas medicinais; Peumus boldus; Medicina tradicional.

\section{ABSTRACT}

The practices with medicinal plants are popular all over the world, in some places they are used more frequently, especially when it comes to the culture of a certain community. Peumus boldus, or boldo as it is popularly known, is commonly found in home backyards or even in industrialized form. This work is a literature review, carried out from August to November 2019. In which the use of boldo and its benefits and problems as a therapeutic alternative was observed. The research searched for studies published from 2009 to 2019, having as inclusion criteria original articles, only in Portuguese, that presented studies with the boldo of the species Peumus boldus, that talked about the use of plants and 
for what therapeutic purpose. Peumus boldus has a prominent position, related to the predominance of use, and that, when consumed incorrectly, can cause fetal malformation, diarrhea, among others. However, when consumed correctly, it brings many benefits for some gastrointestinal changes, such as acting as an antiflatulent, hepatoprotective, digestive, etc. Therefore, it is important for the population to be aware of the benefits and problems of using medicinal plants, especially boldo, by the population.

Descriptors: Medicinal plants; Peumus boldus; Traditional medicine.

\section{INTRODUÇÁO}

O emprego das plantas medicinais, no cuidado à saúde, está presente na sociedade há muitos anos. Há indícios da utilizaçáo de recursos naturais nas civilizaçôes mais antigas, sendo o consumo por via oral, em forma de chá, um dos mais referidos na literatura, com seus primeiros usos no século 27 a.C. ${ }^{1,2}$.

Devido aos seus efeitos benéficos e por ser importante fonte de compostos ativos, as plantas permanecem como alternativa terapêutica na prevenção e tratamento de doenças ${ }^{3}$. O principal motivo é a facilidade de acesso e o valor reduzido, quando comparados aos medicamentos sintéticos ${ }^{4}$

$\mathrm{Na}$ perspectiva de fortalecimento e necessidade de normatização do uso das plantas medicinais na atenção primária surgem em 2006, a Política Nacional de Práticas Integrativas Complementares (PNPIC) e o Programa Nacional de Plantas Medicinais e Fitoterápicos. Esses têm como objetivo garantir à população brasileira o acesso seguro e o uso racional de plantas medicinais, estabelecendo um impulso às práticas populares e tradicionais do uso de plantas medicinais e remédios caseiros, já conhecidos pelos diversos povos do Brasil ${ }^{5}$.

Uma das plantas medicinais de amplo conhecimento e uso popular é o boldo (Peumus boldus). O boldo é utilizado principalmente para o tratamento de distúrbios gastrointestinais, agindo como hepatoprotetor, digestivo, laxante suave, anti-espasmódico, antiflatulento e diurético. Porém, além de suas açôes positivas, o boldo não deve ser utilizado de forma continua e por períodos longos, pois em altas doses pode acarretar em vômito, diarreia, efeitos narcóticos e até convulsão. Ainda, é contraindicado em casos específicos, como por exemplo, obstrução de vias biliares ${ }^{8,9}$.

Dessa forma, é importante que os profissionais da saúde, estejam preparados para atender os usuários e orientar sobre uso correto das plantas medicinais. Sendo assim, o presente estudo tem por objetivo descrever sobre os benefícios e os problemas relacionados ao uso da forma incorreta de Peumus boldus. 


\section{METODOLOGIA}

Trata-se de uma revisão bibliográfica, realizada no período de agosto a novembro de 2019. A pesquisa buscou por estudos publicados do ano de 2009 à 2019, tendo como critérios de inclusão artigos originais, apenas na língua portuguesa, que apresentassem estudos com o boldo da espécie Peumus boldus, que falassem sobre a utilização de plantas e para qual finalidade terapêutica. Constituíram-se como critérios de exclusão artigos em que não foi possível identificar uma relação com a temática por meio da leitura do título e resumo, artigos que se repetiam nas bases de dados, artigos de revisão e capítulos de livros.

As pesquisas foram realizadas nas bases de dados Literatura Latino-Americana e do Caribe em Ciências da Saúde (LILACS), Literatura Internacional em Ciências da Saúde (MEDLINE), Scientific Electronic Library Online (SciELO), e os descritores utilizados foram: Plantas medicinais; Peumus boldus; Medicina tradicional. Desta busca foram encontrados 648 artigos, porém, foram utilizados apenas 5 artigos que se encaixaram nos critérios de inclusão.

\section{RESULTADOS E DISCUSSÃO}

Durante a pesquisa foram encontrados 648 artigos. Excluíram-se 643 artigos por não se encaixarem nos critérios de inclusão. Após a análise dos artigos, seguindo os critérios de inclusão e exclusão, restaram apenas 5 artigos que contemplavam o tema a ser abordado nessa revisão bibliográfica. Identificou-se a partir das buscas, que existem poucos estudos com a temática proposta.

$\mathrm{O}$ uso das plantas medicinais reflete a realidade de parte da população, a qual encontra limitaçóes de acesso aos serviços essenciais de saúde. Além desse, o conhecimento popular acerca da finalidade terapêutica das plantas medicinais favorece o uso destas ${ }^{10}$. Em um estudo realizado com moradores da comunidade rural de Santo Antônio de Jesus na Bahia 53,6\% utilizavam apenas remédios caseiros, dentre esses as plantas medicinais, como métodos de tratamento, sendo que $94,6 \%$ cultivavam a própria planta no quintal de casa ${ }^{11}$.

Ainda, segundo o estudo a prevalência do uso, isolado ou em associação com medicamentos, de plantas medicinais em uma unidade básica de saúde da família foi de 79\%, comprovando o amplo uso das plantas como forma de tratamento. $\mathrm{O}$ mesmo estudo evidenciou a prevalência do sexo feminino no uso desta prática complementar, em um total de 420 participantes, $78 \%$ dos que usavam plantas eram mulheres ${ }^{12}$.

A maior predominância por parte do sexo feminino no uso das plantas medicinais pode ser explicada pelo fato das mulheres preocuparem-se mais com a saúde quando comparadas com populaçóes do sexo masculino. Bem como, serem as maiores responsáveis por passar para as futuras geraçôes da família os conhecimentos sobre as ervas medicinais ${ }^{13}$. 
Dentre as plantas mencionadas com mais frequência nos estudos realizados em Estratégias Saúde da Família e em domicílios, o boldo merece destaque, foram entrevistadas 154 pessoas com o objetivo de identificar quais as plantas em uso pela população rural, e o boldo foi uma das plantas mais citadas ${ }^{11}$. Em outra pesquisa, foi escolhido o Mercadão de Madureira no estado do Rio de Janeiro para a realização de busca ativa das plantas mais comercializadas para o uso medicinal e, das 20 plantas mais compradas, o boldo é o mais procurado ${ }^{14}$.

Conforme o estudo realizado na Paraíba, o boldo está em primeiro lugar de uso, sendo utilizado pela população para digestão. Os mais de 400 entrevistados ainda relataram que a parte mais utilizada do boldo é o caule junto com as folhas, e a forma de preparo é por infusão, sua frequência de uso duas vezes ao dia quando necessário e a forma de consumo é feita por via oral ${ }^{12}$.

Acredita-se que a prevalência do consumo do boldo é devida sua ação direta e rápida, ou seja, apresenta uma resposta efetiva e ágil frente ao problema. Em todos os estudos de base populacional pesquisados, os entrevistados relataram fazer o uso da planta para dores no estômago e problemas relacionados à digestão após as refeiçóes. Ainda, foi citado o uso da planta para constipação e flatulência ${ }^{11}$.

As atividades farmacológicas do boldo são atribuídas a sua ação coletérica e colagoga, ou seja, estímulo da produção da bile e secreção da bile para o duodeno. É estimulante das secreçôes gástricas, facilitando assim a digestão, bem como tem efeito hepatoprotetor, antioxidante, antiinflamatório e diurético. E em associaçóes com outras plantas medicinais como alcachofra (Cynara cardunculus var. scolymus), tem ação em ardores esofágicos e epigástricos, e com cáscara sagrada (Rhamnus purshiana) é usada para constipação ${ }^{8,9}$.

Sua ação terapêutica é oriunda dos metabólitos secundários presentes na planta. Embora na sua constituição haja a presença de metabólitos como flavonoides, óleos essenciais e catequinas, o alcaloide boldina é o principal constituinte ativo ${ }^{8,9}$.

A boldina é um alcaloide encontrado tanto nas cascas como nas folhas do boldo, sua concentração nas cascas de até $6 \%$ e nas folhas é em torno de $0,14 \%$. Muitas atividades farmacológicas têm sido atribuídas ao alcaloide puro, como relaxante da musculatura lisa intestinal, atividade tripanossomicida in vitro e efeito imunomodulador in vitro para células isoladas de pacientes com leucemia linfocítica crônica ${ }^{15}$. Os imunomoduladores são terapias imunológicas recentes, sendo drogas que podem exacerbar ou reduzir resposta imune, para tratamento de doenças causadas por hipersensibilidades ou imunodeficiências ${ }^{16}$.

Foram encontrados efeitos anticonvulsivante e antinociceptivo, devido a presença da boldina, medidos via bloqueio de receptores dopaminérgicos D2. A literatura demonstrou muitos estudos que evidenciam o potencial antioxidante do boldo. São encontrados também nessa planta, devido à presença do alcaloide boldina, atividades farmacológicas antitumorais, citoprotetora, anti-inflamatória e antipirética, que são propriedades sequestradoras de radicais livres desta molécula ${ }^{15}$. 
Embora haja comprovação científica dos benefícios do uso do boldo, devido seus metabólitos secundários, há também, muitas vezes desconhecido pela população, problemas relacionados ao seu uso. Em grandes quantidades o boldo pode ser hepatotóxico e causar má formação fetal. Um estudo com extrato hidroalcóolico de boldo em ratas prenhas demonstrou alteraçóes anatômicas no feto e alteraçóes histológicas no fígado dos ratos quando usado $800 \mathrm{mg} /$ $\mathrm{kg}$ da planta. De acordo com a literatura, todas estas espécies de boldo possuem propriedades abortivas confirmadas ${ }^{17}$.

Em um estudo realizado em uma Unidade Básica de Saúde de São Paulo, com 48 mulheres, incluindo mulheres no período gestacional, novamente o boldo (peumus boldus) teve destaque com (42\%), entre as plantas medicinais mais consumidas e citadas pelas entrevistadas. Demonstrando que, ainda há falta de informação a respeito de potenciais problemas teratogênicos causados pelo uso da planta ${ }^{18}$.

Quando questionados sobre a indicação da planta, alguns estudos citam que a população faz uso por indicação de um não profissional da saúde. Relatou-se na pesquisa que a indicação de uso se deu principalmente por parentes (84\%), seguidos por amigos (9\%), meios de comunicação (televisão - 2,6\%), médico $(1,5 \%)$ e revista $(1,5 \%)$. Ainda, o estudo supracitado demonstra que foi observado que a maioria dos participantes não recebeu informaçôes de profissionais capacitados $^{12}$.

$\mathrm{O}$ uso de plantas medicinais se tornou um costume, que ao longo dos anos não se perdeu, o Ministério da Saúde estimula a introdução desta prática como terapia complementar no Sistema Único de Saúde (SUS). Porém, como todos os chás, o boldo deve ser consumido de forma moderada, pois há muitos estudos que comprovam que o uso por tempo prolongado e em altas doses pode ser tóxico. A atenção deve ser redobrada, principalmente, para gestantes ${ }^{19}$.

Por conta disso, faz-se necessário que os profissionais da saúde, em especial os enfermeiros que atuam no cuidado ao paciente, busquem ter conhecimentos relacionados não somente aos benefícios do boldo, como também as possíveis açôes maléficas, para que possam oferecer uma assistência à saúde mais completa aos indivíduos, unindo, assim, conhecimento popular e científico ${ }^{13}$.

\section{CONCLUSÓES}

Este estudo evidenciou que, a maioria da população faz uso de plantas medicinais devido à facilidade de acesso, ao baixo custo e pelo conhecimento popular. Dentre as plantas mais procuradas e utilizadas está Peumus boldus, conhecido popularmente como boldo, sendo utilizado principalmente para alteraçóes gastrointestinais.

Embora seja benéfico, as pessoas fazem o uso do boldo sem os devidos conhecimentos das possíveis complicações, e efeitos indesejados que essa planta pode trazer. Dessa forma, é importante que sejam realizadas mais pesquisas científicas neste campo, e que os profissionais 
da saúde possam ter um conhecimento técnico-científico mais concreto e ampliado, para então orientar adequadamente a população quanto a sua utilização.

\section{REFERENCIAS}

1. Lima, RFDS et al. Práticas populares de cura e o uso de plantas medicinais por mães ribeirinhas no cuidado infantil. RevFundCare Online [Internet]. Dezembro de 2017 [citado em 28 de agosto de 2019]; 9;(4)(1155) Rio de Janeiro. Disponível em: http:// www.seer.unirio.br/index.php/cuidadofundamental/article/download/5982/pdf_1.

2. Morais SM, Cavalcanti ESB, Costa SMO, Aguiar LA. Ação antioxidante de chás e condimentos de grande consumo no Brasil. Rev. bras. farmacogn. [Internet]. Março de 2009 [citado em 18 de outubro de 2019]; 19(1b):315-320. Disponível em: http://www. scielo.br/scielo.php?script=sci_arttext\&pid=S0102-695X2009000200023\&lng=en. http://dx.doi.org/10.1590/S0102-695X2009000200023.

3.Cidade De São Paulo Subprefeituras. Medicinas Tradicionais. Disponível em: https:// www.prefeitura.sp.gov.br/cidade/secretarias/subprefeituras/noticias/?p=20386.

4. Badke MR, Budó MLD, Alvim NAT, Zanetti, GD, Heisler EV. Saberes e práticas populares de cuidado em saúde com o uso de plantas medicinais. Texto contexto - enferm. [Internet]. Junho de 2012 [citado em 16 de outubro de 2019]; 21(2):363-370. Disponível em: http://www.scielo.br/scielo.php?script=sci_ arttext\&pid=S0104-07072012000200014\&lng=en. http://dx.doi.org/10.1590/S010407072012000200014.

5. Ministério Da Saúde. Política e Programa Nacional de Plantas Medicinais e Fitoterápicos. Disponível em: http://www.saude.gov.br/acoes-e-programas/programanacional-de-plantas-medicinais-e-fitoterapicos-ppnpmf/politica-e-programa-nacionalde-plantas-medicinais-e-fitoterapicos.

8. Santos, LA et al. Determinaçao da Atividade Antimicrobiana do Extrato Hidroalcoólico da Planta PlectranthusornatusCodd (Boldo Chinês). Rev Med Minas Gerais. 2014 [citado em 16 de outubro de 2019]; 24(4):464-469. Disponível em: http://www.rmmg.org/artigo/detalhes/1705.

9. Félix-Silva J, Tomaz IM, Silva MG, Santos KSCR, Silva-Júnior AA, Carvalho MCRD. Identificação botânica e química de espécies vegetais de uso popular no Rio Grande do Norte, Brasil. Rev. bras. plantas med. [Internet]. 2012 [16 de outubro de 2019]; 14(3):548-555. Disponível em: http://www.scielo.br/scielo.php?script=sci_ arttext\&pid=S1516-05722012000300018\&lng=en.

10. Santos MRA; Lima MR; Ferreira MGR. Uso de plantas medicinais pela população de Ariquemes, em Rondônia. Hotic. Bras. 2008 [citado em 24 de novembro de 2019] 26: 244-250. Disponível em: http://www.scielo.br/pdf/hb/v26n2/23.pdf. 
11. Santos RS et al. Uso regular de plantas medicinais para fins terapêuticos em famílias residentes na zona rural de Santo Antônio de Jesus - Bahia - Brasil. J. Health Biol Sci [Internet]. 2017 [citado em 5 de novembro de 2019]; 5(4):364-370. Disponível em: https://periodicos.unichristus.edu.br/jhbs/article/view/1317.

12. Araújo CRFD et al. Perfil e prevalência de uso de plantas medicinais em uma unidade básica de saúde da família em Campina Grande, Paraíba, Brasil. Ver. ciênc. farm. básica apl. [Internet] 2014 [citado em 6 de novembro de 2019]; 35(2):233-238. Disponível em: https://pesquisa.bvsalud.org/portal/resource/pt/lil-737341.

13.Ceolin T, Heck RM, Barbieri RL, Schwartz E, Muniz RM, Pillon CN. Plantas medicinais: transmissão do conhecimento nas famílias de agricultores de base ecológica no Sul do RS. Rev. esc. enferm. USP [Internet]. Março de 2011 [citado em 18 de novembro de 2019]; 45(1):47-54. Disponível em: http://www.scielo.br/scielo. php?script=sci_arttext\&pid=S0080-62342011000100007\&lng=pt. http://dx.doi. org/10.1590/S0080-62342011000100007.

14. Bochner R, Fiszon JT, Assis MA, Avelar KES. Problemas associados ao uso de plantas medicinais comercializadas no Mercadão de Madureira, município do Rio de Janeiro, Brasil. Rev. bras. plantas med. [Internet]. 2012 [citado em 6 de novembro de 2019];14(3):537-547. Disponível em: http://www.scielo.br/scielo.php?script=sci_ arttext\&pid=S1516-05722012000300017\&lng=en. http://dx.doi.org/10.1590/S151605722012000300017.

15. Schwanz M et al. Caracterização Farmacobotânica de Peumus boldus (Monimiaceae) e Avaliação de Atividades Biológicas do Alcalóide Boldina. Lat. Am. J. Pharm [Internet]. 2008 [citado em 24 de novembro de 2019]; 27(6): 871-9.

Disponível em: http://www.latamjpharm.org/trabajos/27/6/LAJOP_27_6_1_11_ OGM187JM50.pdf.

16. Lima, HC. Imunologia clínica dos imunomoduladores: Tópicos em Imunodermatologia Clínica. 1. ed. São Paulo: Segmento Farma, 2004. p. 121-32.

17. Almeida ERD, Melo AM, Xavier H. Avaliaçáo toxicológica do extrato hidroalcoólico das folhas secas de Peumus boldus e boldine em ratos: subtítulo do artigo. Wiley online library: Phytotherapy Research, Local [Internet]. 2000 [citado em 24 de novembro de 2019]; 14(2):99-102. Disponível em: https://onlinelibrary. wiley.com/doi/abs/10.1002/(SICI)1099-1573(200003) 14:2\%3C99::AIDPTR600\%3E3.0.CO;2-4.

18. Souza MNCV, Tangerina MMP, Silva VC, Vilegas W, Sannomiya M. Plantas medicinais abortivas utilizadas por mulheres de UBS: etnofarmacologia e análises cromatográficas por CCD e CLAE. Rev. bras. plantas med. [Internet]. 2013 [citado em 6 de novembro de 2019.]; 15(4):763-773. Disponível em: http://www.scielo.br/ scielo.php?script=sci_arttext\&pid=S151605722013000500018\&lng=en. 
19. Viegas CF, Franciscon CH, Lima MP. Abordagem Etnobotânica em uma empresa do Polo Industrial de Manaus. Scientia Amazonia. 2014 [citado em 18 de novembro de 2019]; 3(3):51-58. Disponível em: http://scientia-amazonia.org/wp-content/ uploads/2016/06/v3-n3-51-58-2014.pdf.

Autor Correspondente: Maísa Schneider Lazarotto E-mail: maisaslazarotto@gmail.com Recebido em: 2021-05-24 Aprovado em: 2021-06-22 